\title{
FILTRATION TECHNIQUES IN THE STUDY OF LIE ALGEBRAS
}

\author{
BY BRIAN T. SMITH
}

Communicated by George B. Seligman, September 18, 1972

1. Introduction. In the present note we announce some results which have been obtained by the systematic exploitation of filtration arguments in the study of (infinite dimensional) Lie algebras. The fundamental theorem along this line appears in the following section. In $\S 3$ we indicate how this result can be used to provide a new proof and at the same time a generalization of the Shirshov-Witt subalgebra theorem; we recall that this theorem asserts that, with ground ring a field, a subalgebra of a free Lie algebra is free (this being of course the Lie-theoretic analogue of the Nielsen-Schreier subgroup theorem). $\$ 4$ contains the statement of a result which will be seen to be the coproduct analogue of M. Hall's basis theorem for a free Lie algebra. From the existence of such a coproduct basis one is easily led to examples which show that there is no (at least strict) Lie-theoretic analogue of the Kurosch subgroup theorem; the problem of the structure of the subalgebras of a coproduct of Lie algebras takes on added interest. Some results along this line are listed in $\S 5$; this section contains also a criterion for the freeness of a Lie algebra. The sixth and final section contains acknowledgments and remarks.

2. Setting of the context and statement of the fundamental theorem. It is to be expected that as we employ filtration arguments we should eventually find ourselves dealing with graded objects. In order to unify the presentation we have elected to assume from the beginning that our objects are graded. We let $J$ denote a monoid whose operation is written additively and whose set of nonzero elements is denoted by $J^{o}$, and $R$ a commutative ring with unit. The notions of $J$-graded $R$-module, $J$-graded $R$-algebras, etc. are defined in the usual way. The sign commutation rule requires that the notion of odd and even be defined for the elements of $J$. We are led in this way to the notion of an oriented monoid: An oriented monoid $J$ is a pair consisting of a commutative monoid, also denoted by $J$, with the cancellation property and a morphism $\varepsilon: J \rightarrow Z / 2 Z$ of monoids, where $\boldsymbol{Z}$ denotes the monoid of rational integers. The morphism $\varepsilon$ is called the orientation of $J$; an element $s$ in $J$ is called even if $\varepsilon(s)=0$ and odd if

AMS(MOS) subject classifications (1970). Primary 17B65; Secondary 17B35, $17 \mathrm{~B} 55$.

Key words and phrases. Lie algebra, universal enveloping algebra, filtered algebra, associated graded functor, free Lie algebra, coproduct of Lie algebras, standard monomial. 
$\varepsilon(s)=1$; the monoid $J$ is called even if all of its elements are even. When the monoid is even the sign commutation rule can be effectively ignored. For the remainder of this note $J$ denotes an oriented monoid.

The standard concepts of homological algebra readily extend to the context of objects graded on an oriented monoid. There is gain to be had by requiring that the monoid be divergent; by definition a divergent monoid is an oriented monoid $J$ such that $J^{o}$ is closed under the operation of addition and the intersection $\bigcap_{1 \leqq k} \varphi_{k}\left(\left(J^{o}\right)^{k}\right)$ is empty, where $\varphi_{k}: J^{k} \rightarrow J$ is the $k$-fold addition function. Homological algebra for objects graded on divergent monoids presents some very agreeable aspects; we mention two instances, both having to do with tensor algebras and both being destined to play crucial roles in our arguments. Before doing this we need to recall that a $J$-graded $R$-algebra is said to be connected if $\eta(A): R \rightarrow A_{0}$ is an isomorphism, where $\eta(A): R \rightarrow A$ denotes the unit morphism of the algebra $A$. Thus, for example, a tensor algebra $T(X)$ on a graded module $X$ is connected if $X_{0}=0$. Now with a little effort the following two results can be established: if $T(X)$ is a connected tensor algebra graded on a divergent monoid over $R$ and $X$ is projective over $R$ (this just means that, for each $s$ in $J, X_{s}$ is projective over $R$ in the usual, that is, ungraded, sense) then any $T(X)$-module $Z$ which is projective over $R$ has projective dimension $\leqq 1$; suppose that $B$ is a connected algebra graded on a divergent monoid over $R$ such that the projective dimension of $R$ over $B$ is $\leqq 1$; then, if $A$ is a subalgebra of $B$ such that $B$ is projective over $A, A$ is isomorphic with the tensor algebra $T(Q(A)), Q(A)$ is projective over $R$ and the projective dimension of $R$ over $A$ is $\leqq 1$. We recall that for a supplemented algebra $A$ the $R$-module $Q(A)$ of the indecomposable elements of $A$ is defined to be the cokernel of the natural morphism $I(A) \otimes I(A) \rightarrow I(A)$, where $I(A)$ denotes the kernel of the augmentation morphism $\varepsilon(A): A \rightarrow R$.

Recall that a filtration on a $J$-graded $R$-module $X$ is an increasing sequence $F_{p} X$ of submodules of $X$ with $p$ running through $Z$. A filtration $\left(F_{p} X\right)$ on $X$ is said to be nonnegative if $F_{p} X=0$ for $p<0$ and to be cocomplete if $\bigcup_{p} F_{p} X=X$. Noting that each $F_{p} X$ is itself a $J$-graded $R$-module and that $F_{p-1} X$ may be regarded as a submodule of $F_{p} X$, we may define a $Z$-graded object $E^{0}(X)$ by setting $E_{p}^{0}(X)$ equal to the factor module $F_{p} X / F_{p-1} X$. Defining morphisms of filtered modules in the usual way, we see that $E^{0}$ may be regarded as a functor from the category of filtered $J$-graded $R$-modules to the category of $\boldsymbol{Z} \times J$-graded $R$-modules; $\boldsymbol{Z} \times J$ represents the product monoid of the monoids $\boldsymbol{Z}$ and $J$ which for our purposes may be regarded as oriented by giving it the even orientation. But observe that this product monoid will not be divergent; however the filtrations with which we shall be concerned will all have the property that the corresponding associated graded objects may be regarded as 
graded on the submonoid $\left(\boldsymbol{Z}^{+} \times J\right) \cup\{(0,0)\}$ which is divergent, where $\boldsymbol{Z}^{+}$denotes the set of positive integers. It is clear what is to be understood by a filtration on an algebra being compatible with the underlying algebra structure; proceeding from such a filtration, we can define on the corresponding associated graded object the same type of algebra structure; thus, for example, if $A$ is a supplemented $J$-graded $R$-algebra with a filtration defined on it which is compatible with its supplemented algebra structure, then $E^{0}(A)$ may in a natural way be regarded as a supplemented $R$-algebra (graded on a suitable monoid).

THEOREM A. Let $J$ denote an even divergent monoid and let L denote a $J$-graded Lie algebra over $R$. Suppose that $L$ injects via the canonical morphism into its universal enveloping algebra $U(L)$ and $U(L)$ has defined on it a filtration which is compatible with its supplemented algebra structure, cocomplete, nonnegative and $F_{p} U(L)=R$ for $p=0$; moreover the canonical morphism $U\left(E^{0} L\right) \rightarrow E^{0}(U L)$ is an isomorphism and $U\left(E^{0} L\right)$ is a connected tensor algebra on a projective module. Then $L$ is a projective Lie algebra.

In the preceding $L$ inherits the filtration from $U(L)$; it is trivial to check that a filtration which is compatible with the supplemented algebra structure of $U(L)$ will be compatible with the associated Lie algebra structure of $U(L)$; hence the induced filtration on $L$ will be compatible with its Lie algebra structure. Thus $E^{0}(L)$ may be regarded as a Lie algebra. Since the filtration-preserving morphism $L \rightarrow U(L)$ induces a morphism $E^{0}(L) \rightarrow E^{0}(U L)$ on the $E^{0}$-level, there exists by the fundamental property of the universal enveloping algebra a unique morphism $U\left(E^{0} L\right) \rightarrow E^{0}(U L)$ making the usual diagram commutative. A projective Lie algebra is the Lie subalgebra of a tensor algebra $T(X)$ on a projective module $X$ generated by $X$; denoting such a Lie algebra by $L(X)$ we have that $L(X)$ is free (on $X$ ) when $X$ is free (as module).

The proof of the theorem is surprisingly straightforward. Using the two relations $Q(U L)=L /[L, L]$ valid for any Lie algebra $L$ and $Q(T X)=$ $X$ valid for any module $X$, we get from the last hypothesis of the theorem that $E^{0}(L) /\left[E^{0}(L), E^{0}(L)\right]$ is $R$-projective; the projectiveness of this factor module allows us to pick a submodule of $L$ which is itself projective and which by its very choice forms a set of generators of $L$. The verification that $L$ is in fact "projectively" generated by this submodule is relatively routine.

3. The subalgebra theorem. Our generalization of the Shirshov-Witt theorem is

THEOREM B. Let $L(X)$ be a projective Lie algebra which is filtered as a subalgebra of the canonically filtered algebra $U(L X)=T(X)\left(F_{p} T(X)=\right.$ $R \coprod X \coprod \cdots \coprod X^{p \otimes}$ where $X^{p \otimes}=X \otimes \cdots \otimes X$ denotes the p-fold 
tensor product of $X)$. Suppose that $L^{\prime}$ is a subalgebra of $L(X)$ such that the monomorphism $E^{0}\left(L^{\prime}\right) \rightarrow E^{0}(L X)$ is split over $R$. Then $L^{\prime}$ is a projective Lie algebra.

An immediate corollary of this result is the Shirshov-Witt subalgebra theorem. In the statement of the preceding theorem it is understood that the underlying monoid is even and divergent; observe that the usual type of (ungraded) Lie algebra can always be regarded as trivially graded. We indicate now very briefly how the preceding theorem follows from Theorem A: A twofold extension of Proposition XIII, 4.1 in Cartan and Eilenberg [1] enables us to conclude that the natural morphism $U\left(E^{0} L^{\prime}\right) \rightarrow$ $U\left(E^{0}(L X)\right)$ is a monomorphism and that the latter module is projective as a module over the former (on either side). Using the easily established relation $E^{0}(L X)=L\left(E^{0} X\right)$, we get that $U\left(E^{0}(L X)\right)$ is a connected tensor algebra on a projective module (namely on $E^{0}(X)$ ). Making use of this as well as of those special properties of connected tensor algebras graded on a divergent monoid mentioned in the preceding section, we are able to conclude that $U\left(E^{0} L^{\prime}\right)$ is a connected tensor algebra on a projective module. The only other hypothesis of Theorem $A$ that needs any verifying at all is that the morphism $U\left(E^{0} L^{\prime}\right) \rightarrow E^{0}\left(U L^{\prime}\right)$ is in fact an isomorphism. The filtration on $U\left(L^{\prime}\right)$ can be defined in such a way (namely as a certain one induced by the filtration on $L^{\prime}$ ) that the surjectivity of the morphism in question is immediate; the injectivity can be established with the help of the following commutative diagram

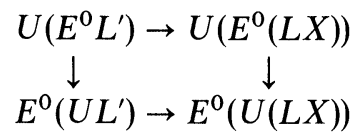

in which - as we have already seen - the top horizontal map is injective. Hence it suffices to show that the right vertical map is injective; that it is indeed bijective follows from the relations

$$
U\left(E^{0}(L X)\right)=U\left(L\left(E^{0} X\right)\right)=T\left(E^{0} X\right)=E^{0}(T X)=E^{0}(U(L X))
$$

all of which can be straightforwardly verified.

4. A coproduct basis. In this and the following section the underlying monoid is taken to be even and divergent. Letting $A$ denote an indexing set we assume that, for each $\alpha$ in $A, L_{\alpha}$ is a Lie algebra which is $R$-free, say with basis denoted by $X_{\alpha}$. We emphasize that each $X_{\alpha}$ is a graded set. Setting $X$ equal to the (disjoint) union of the $X_{\alpha}$ we consider the nonassociative words formed from the elements of $X$; any such word $w$ has associated with it in a unique way a length which we shall denote by 
$l(w)$. The words of length 1 are of course just the elements of $X$; for $x$ in $X$ we shall denote by $|x|$ the unique index $\alpha$ for which $x$ lies in $X_{\alpha}$. We define now various subsets of these nonassociative words each of which will be called a set of standard monomials (in $X$ ). The words of length 1 are the standard monomials of length 1 and are simply ordered by first simply ordering the indexing set $A$ and each set $X_{\alpha}$ and then letting $X$ have the resulting induced simple ordering. The standard monomials of length 2 are defined to be those words of length 2 of the form $x^{\prime} x^{\prime \prime}$ for which $\left|x^{\prime}\right|>\left|x^{\prime \prime}\right|$; these are simply ordered; by requiring that any standard monomial of length 1 is less than any standard monomial of length 2 we obtain a simple ordering of the standard monomials of length $\leqq 2$. We assume that the standard monomials of length $<n(n>2)$ have been defined from the subset of words of length $<n$ and simply ordered in such a way that for standard monomials $r$ and $s, l(r)<l(s)$ implies that $r<s$. Then a word $w$ of length $n$ is said to be a standard monomial if the following conditions are satisfied:

(i) $w=u v$ where $u$ and $v$ are standard monomials and $u>v$;

(ii) [by (i) and the induction assumption it follows that $l(u)>1$ ] writing $u=u_{1} u_{2}$, we have that $u_{2} \leqq v$;

(iii) if $l(v)=1$ so that-as is readily seen-u must be of the form $\left(\cdots\left(x x_{1}\right) \cdots\right) x_{k}$ where $x, x_{1}, \cdots, x_{k}$ are all in $X$, then $x \leqq v$ if $|x|=|v|$.

The standard monomials of length $n$ are simply ordered; by requiring that any standard monomial of length $<n$ is less than any standard monomial of length $n$ we obtain a simple ordering of the standard monomials of length $\leqq n$. The definition is now completed by induction.

Writing the Lie product by juxtaposition, we may regard the nonassociative words and hence the standard monomials in $X$ as a subset of the coproduct $\bigsqcup L_{\alpha}$ of the $L_{\alpha}$. When each $X_{\alpha}$ consists of a single element the corresponding coproduct may be identified with the free Lie algebra on $X$; our standard monomials are then just the standard monomials of Marshall Hall, Jr. [2]. The following theorem is the coproduct analogue of Hall's basis theorem.

THEOREM C. Suppose that $L_{\alpha}, \alpha$ in A, is a family of Lie algebras and that each $L_{\alpha}$ is $R$-free. Then the coproduct $\amalg L_{\alpha}$ of the $L_{\alpha}$ is $R$-free; indeed if $X_{\alpha}$ denotes an $R$-basis of $L_{\alpha}$ then any set of standard monomials defined from the union of the $X_{\alpha}$ constitutes an R-basis of $\coprod L_{\alpha}$.

A straightforward albeit laborious argument which is nothing more than Hall's own argument in a coproduct garb serves to establish the preceding result. However an appeal to filtration techniques allows us to reduce the proof of the theorem to the special case in which the $L_{\alpha}$ are all abelian. 
5. The structure of various subalgebras of the coproduct. We shall merely list here a number of results which we have obtained by using the coproduct basis theorem of the preceding section and/or filtration techniques.

THEOREM D. Let $L_{\alpha}, \alpha$ in $A$, be a family of Lie algebras and suppose that each $L_{\alpha}$ is free as $R$-module. Then the kernel of the natural morphism of the coproduct of the $L_{\alpha}$ into the corresponding product is a free Lie algebra; if $D$ denotes this kernel, there is the direct sum decomposition $\bigsqcup L_{\alpha}=$ $\sum L_{\alpha} \oplus D$. If $A$ has been simply ordered in such a way that there exists an $\alpha_{0}$ in $A$ such that for $\alpha>\alpha_{0}, L_{\alpha}$ is 1-dimensional, then the subalgebra generated by the $L_{\alpha}, \alpha>\alpha_{0}$, and $D$ is free, and is equal to the direct sum

$$
\sum_{\alpha>\alpha_{0}} L_{\alpha} \oplus D \text {. }
$$

Here $\sum L_{\alpha}$, resp. $\sum_{\alpha>\alpha_{0}} L_{\alpha}$, denotes the subspace of the coproduct generated by the $L_{\alpha}, \alpha$ in $A$, resp. $\alpha>\alpha_{0}$.

THEOREM E. With the ground ring assumed to be semisimple, the kernel of the unique morphism $L \amalg L \rightarrow L$ which induces the identity morphism on each factor $L$ of $L \coprod L$ is a projective Lie algebra.

THEOREM F. With the ground ring assumed to be semisimple, for any Lie algebra $L$ the pullback of the diagram

$$
L \prod_{L \rightarrow L} L
$$

is a projective Lie algebra.

COROLlARY. With the ground ring assumed to be semisimple, a Lie algebra $L$ is projective if and only if there exists a morphism $L \rightarrow L \coprod L$ making the following diagram commutative

$$
\underset{\nearrow}{L \prod_{\downarrow} L} L
$$

In both of the preceding results $L \rightarrow L \prod L$ denotes the diagonal morphism and $L \coprod L \rightarrow L \prod L$ the unique morphism which takes the left resp. righthand factor $L$ of $L \coprod L$ into the corresponding factor of the product $L \prod L$.

THEOREM G. Let $L_{\alpha}$, $\alpha$ in $A$, be Lie algebras over a field and suppose that $L$ is a subalgebra of $\coprod L_{\alpha}$ intersecting $\sum L_{\alpha}$ in the zero subspace. Then $L$ is free.

6. Acknowledgments and comments. The results announced in this note 
are contained in the author's doctoral dissertation written under the direction of Professor John C. Moore at Princeton University; the author takes this opportunity to offer his profound thanks and gratitude to Professor Moore for his invaluable assistance, advice and encouragement; the idea of exploiting filtration arguments in the study of Lie algebras as outlined in the preceding originated with him. The Shirshov-Witt theorem appears in Shirshov [4] and Witt [6]. Hall's basis theorem [2] was the inspiration for our Theorem C; Shirshov [5] has also obtained a coproduct basis. Theorems D and $\mathrm{G}$ have also appeared in Kukin [3]. The corollary of Theorem $\mathrm{F}$ is the analogue of a well-known criterion for the freeness of a group; a straightforward proof of this group-theoretic criterion makes use of the Kurosch subgroup theorem which has no analogue for Lie algebras. I know at the present no proof other than a filtration one for this Lie-theoretic criterion.

Full details of these as well as of other results are to appear in a forthcoming paper of the author.

\section{REFERENCES}

1. H. Cartan and S. Eilenberg, Homological algebra, Princeton Univ. Press, Princeton, N.J., 1956. MR 17, 1040.

2. Marshall Hall, Jr., A basis for free Lie rings and higher commutators in free groups, Proc. Amer. Math. Soc. 1 (1950), 575-581. MR 12, 388.

3. G. Kukin, Concerning the Cartesian subalgebra of the coproduct of Lie algebras, Algebra i Logika 9 (1970), no. 6, 701-713. (Russian)

4. A. Siršov, Subalgebras of free Lie algebras, Mat. Sb. 33 (75) (1953), 441-452. (Russian) MR 15, 596.

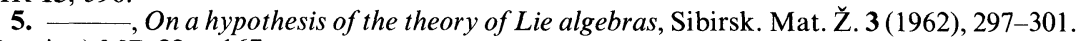
(Russian) MR 32 \# 167.

6. E. Witt, Die Unterringe der freien Lieschen Ringe, Math. Z. 64 (1956), 195-216. MR 17, 1050 . 02115

Department of Mathematics, Northeastern University, Boston, Massachusetts 